\title{
RECHARGE - A Brief Psychological Intervention to Build Resilience in Health Care Workers During the COVID-19 Pandemic: Study Protocol for a Randomized Controlled Trial
}

\section{Naser Morina ( $\square$ naser.morina@usz.ch )}

UniversitätsSpital Zürich: UniversitatsSpital Zurich https://orcid.org/0000-0002-6470-4408

\section{Sonja Weilenmann}

UniversitätsSital Zürich, Universität Zürich

\section{Katie S Dawson}

University of New South Wales School of Psychology

Jutta Ernst

UniversitätsSpital Zürich: UniversitatsSpital Zurich

\section{Zelim Zanitti}

UniversitätsSpital Zürich: UniversitatsSpital Zurich

\section{Roland von Känel}

UniversitätsSpital Zürich: UniversitatsSpital Zurich

\section{Matthis Schick}

UniversitätsSpital Zürich: UniversitatsSpital Zurich

\section{Tobias R Spiller}

UniversitätsSpital Zürich: UniversitatsSpital Zurich

\section{Richard A Bryant}

University of New South Wales School of Psychology

\section{Study protocol}

Keywords: COVID-19, randomised controlled trial, protocol, health care workers, psychological treatment, distress, crisis intervention, pandemic, resilience

Posted Date: March 15th, 2021

DOl: https://doi.org/10.21203/rs.3.rs-212942/v1

License: (c) (1) This work is licensed under a Creative Commons Attribution 4.0 International License. 

RECHARGE - a brief psychological intervention to build resilience in health care workers during the COVID-19 pandemic: study protocol for a randomized controlled trial

Naser Morina ${ }^{1,2^{*}}$, Sonja Weilenmann ${ }^{1,2}$, Katie S. Dawson ${ }^{3}$; Jutta Ernst ${ }^{4}$, Zelim Zanitti ${ }^{1}$, Roland von Känel ${ }^{1,2}$, Matthis Schick ${ }^{1,2}$, Tobias R. Spiller ${ }^{1,2}$, Richard A. Bryant ${ }^{3}$

NM \& SW contributed equally to this paper.

${ }^{1}$ Department of Consultation-Liaison Psychiatry and Psychosomatic Medicine, University Hospital Zurich, University of Zurich, Zurich, Switzerland

${ }^{2}$ Medical Faculty, University of Zurich, Zurich, Switzerland

${ }^{3}$ School of Psychology, University of New South Wales, Sydney, Australia

${ }^{4}$ Centre of Clinical Nursing Science, University Hospital Zurich, University of Zurich, Zurich, Switzerland

*Corresponding author:

Naser Morina, PhD, Department of Consultation-Liaison Psychiatry and Psychosomatic Medicine, University Hospital Zurich, University of Zurich, Culmannstrasse 8, CH-8091 Zurich, Switzerland, naser.morina@usz.ch, Tel +41 442555280 


\section{Abstract}

Background: Health care workers (HCWs) typically face high work demands, which can be exacerbated during crises such as the COVID-19 pandemic. These demands may result in high psychological distress and reduced work performance. Although there are psychological interventions to reduce stress in HCWs under normal working circumstances, no intervention have been specifically developed to addresses stress in the context of public health crises such as the current COVID-19 pandemic. This study aims to evaluate the effectiveness of RECHARGE, a psychological intervention specifically developed for HCWs to reduce distress in HCWs. It is based on a brief crisis intervention of the World Health Organization that teaches basic stress management skills from cognitive behavioural therapy.

Methods: A randomized controlled trial (RCT) will be carried out among 160 physicians, nurses, and other HCWs working in hospitals in Switzerland during COVID-19, who are at least moderately distressed. HCWs will be randomised to RECHARGE $(n=80)$ or active treatment as usual (ATAU) $(n=80)$. Pre-intervention (week 1, T1), post-intervention (week 4, T2) and 2-month follow-up (week 12, T3) assessments include psychological distress as primary outcome, and indicators of mental ill-being (worries, anxiety, depression, burnout, traumatic stress, distress due to perceived ethical dilemma) and work performance as secondary outcomes. These outcomes will be compared between HCWs in the RECHARGE and ATAU groups.

Discussion: RECHARGE is an evidence-informed brief, flexible, easily scalable, fully online psychological program that allows delivery in pandemic conditions, including social isolation. Therefore, this program can serve as a much-needed template for an intervention to reduce stress and enhance work performance in HCWs during the COVID-19 pandemic. If proven effective, RECHARGE may not only be used to reduce elevated stress in HCWs in Switzerland, but also globally. 
26 Key words: COVID-19, randomised controlled trial, protocol, health care workers;

27 psychological treatment; distress, crisis intervention; pandemic; resilience;

28

29 ClinicalTrials.gov NCT04531774. Registered on August 26, 2020.

30 https://clinicaltrials.gov/ct2/show/record/NCT04531774 
32 Working in healthcare is inherently demanding. Many physicians, nurses and other healthcare workers (HCWs) have to work long hours and night shifts, manage high administrative workloads, often experience a poor work-life balance, and face difficult situations with their patients [1]. As a result, HCWs may suffer from psychological distress such as burnout, anxiety, and depression, which are highly prevalent in HCWs, and may show a reduced work performance [1]. During pandemics such as the current SARC-CoV-2 outbreak, also referred to as COVID-19, HCW burden can be exacerbated. In addition to an even higher workload and increased ethical dilemmas in patient care, HCWs have to deal with insufficient resources for personal protective equipment, fear of infection and infecting family members, and with social isolation [2-4]. In a rapid review and meta-analysis on the psychological effects of emerging virus outbreaks on HCWs, Kisely and colleagues reported that working with infected patients is associated with higher levels of psychological distress [2]. Similarly, in a review on the psychological effects of the COVID-19 pandemic on HCWs, Pappa and colleagues found a high prevalence of anxiety, mood, and sleep disorders during the pandemic [3].

Organisational and individual support is key to alleviating stress among HCWs both in times of crises such as pandemics $[2,3,5]$ and under normal working circumstances [6, 7]. Accordingly, several psychosocial interventions to reduce stress in HCWs under normal working circumstances have been tested. Results show that both organisational and individual-level interventions are effective in reducing common mental health symptoms and burnout among physicians $[7,8]$. Among the individual-level interventions, cognitivebehavioural (focusing on stress management) and mindfulness-based programmes were shown to be the most effective ones [6-8]. However, there are hardly any evidence-based interventions that address stress during times of public health crises [9]. 

specifically for HCWs to alleviate stress during pandemics and other public health crises. RECHARGE is an abbreviated and adapted version of Problem Management Plus, an evidence-based intervention from the World Health Organization that helps to cope with stress in the wake of adversity $[10,11]$. As a brief psychological intervention for adults affected by adversity emerging from stress exposure, RECHARGE teaches people welldocumented strategies to manage acute stress. Strategies are based on principles cognitivebehavioural therapy, including psychoeducation, a stress reduction technique, skills to managing worries and problem-solve, behavioural activation with a focus on meaningful activities, and relapse prevention [10]. Because pandemics can trigger problems for HCWs that may be different from normal circumstances (e.g., fear of infecting themselves and others, social or leisure time activities may be restricted), some of the strategies were amended to accommodate the current situation. As a result, RECHARGE is applicable to pandemics and other crises, as well as to normal working circumstances within health care. RECHARGE may be particularly suitable to reduce psychological distress in HCWs in times of crisis because the strategies are easy to learn and implement, transdiagnostic, and address stress in otherwise healthy populations. RECHARGE was designed so that it can be delivered remotely (e.g., via video-conferencing platforms) and by a range of trained personnel, including those with no prior mental health qualifications (notably peers). This approach was undertaken to ensure the intervention is scalable and can also be implemented when time is sparse and personal contacts are restricted due to risk of contagion. To test the potential efficacy of the RECHARGE intervention, a randomized controlled trial (RCT) will be conducted to alleviate psychological distress in HCWs during the COVID-19 pandemic in Switzerland. 
Methods

Aim and design

The primary aim of this study is to evaluate the effectiveness of RECHARGE to reduce distress in HCWs during the COVID-19 pandemic. As secondary aim, the study explores whether RECHARGE reduces symptoms of mental-health condition, and enhances work performance. We will conduct an RCT comparing RECHARGE to active treatment as usual (ATAU) in 160 study participants. Allocation to one of the two arms will be randomized with a parallel assignment on a 1:1 basis. See Figure 1 for an overview of the design.

Due to physical distancing rules and the possibility of home confinement during the COVID19 pandemic, all procedures will be conducted online via Skype for Business. The advantage of remotely-delivered interventions is that they allow for flexibility with regards to when and where sessions can occur. This makes the interventions feasible in the context of quarantine or social isolation, and is also highly flexible in terms of timing of delivery. This is important

94 for HCWs to easily integrate participation into their schedules, even under stressful circumstances such as the pandemic.

\section{Hypotheses}

The primary outcome of this RCT is psychological distress as measured by the Kessler Psychological Distress Scale - K-10 [12, 13] at 2-month follow-up. We hypothesise that HCWs in the intervention condition (RECHARGE) demonstrate a lower level of psychological distress on the K-10 at 2-month follow-up than HCWs in ATAU. The secondary outcomes of this RCT are mental health conditions and work performance. We hypothesise that HCWs in the intervention condition demonstrate lower levels of worry, anxiety, depression, burnout, posttraumatic stress, distress due to perceived ethical dilemmas, 
and impaired work performance at 2-month follow-up than HCWs in ATAU. Furthermore, we will compare psychological distress, mental health conditions, and work performance not only at 2-month follow-up, but also immediately after the intervention and after 6 months.

Participants

110 We will enrol $160 \mathrm{HCWs,} \mathrm{who} \mathrm{for} \mathrm{the} \mathrm{purpose} \mathrm{of} \mathrm{the} \mathrm{study} \mathrm{will} \mathrm{include} \mathrm{those} \mathrm{who} \mathrm{define}$ themselves as HCW (also in training) and work in a healthcare role (e.g. nurses, physicians, etc.). Patient contact is no requirement to participate in this study. Participants will be working in German-speaking cantons in Switzerland who meet the following eligibility

114 criteria:

115 Inclusion criteria:

$-\geq 18$ years of age;

- German-speaking;

- Access to a teleconferencing platform;

- At least a moderate level of distress as defined by a score of $\geq 16$ on the Kessler Psychological Distress Scale [13].

Exclusion criteria:

- Enrolment in a similar intervention (e.g., coaching to reduce stress or enhance wellbeing);

- Currently in psychiatric or psychotherapeutic treatment;

- Currently on sick leave for more than two weeks

128 We calculated our required sample size based on a previous trial with Problem Management 129 Plus [14]. On the premise that this adapted version of Problem Management Plus will operate 
similarly in the current context of COVID-19, we based our sample size calculations on the estimated effect size of Cohen's $d=0.4$. Calculations suggest a minimum total sample size of 130, with 65 participants per group (power $1-\mathrm{b}=0.8, \mathrm{a}=.05,2$-sided). Taking into account an expected $20 \%$ attrition at 2-month follow-up, we aim to include a total number of 160 participants (80 in each condition).

Procedure

137 Participants will be consecutively recruited via (a) (online) advertisements on various

138 platforms, (b) presentations at hospitals (c) local publications (e.g., meetings, newsletters, 139 journals), (d) professional associations, and (e) word-of-mouth recommendations. Potential 140 participants can enlist themselves via the study website, where they are directed to an online questionnaire. After reading our online study information and informed consent form, participants are asked to give informed consent by checking the consent box. Then, participants complete a series of questions screening demographic variables (gender, age,

144 profession and area of work, professional experience, percentage of work) and eligibility 145 criteria.

146 After the study coordinators have checked the eligibility criteria, participants receive a link to

147 complete an online questionnaire assessing distress, worries, anxiety, depression, burnout, 148 posttraumatic stress, distress due to perceived ethical dilemmas, work performance, and 149 additional information (work hours, sleep hours, shift work, COVID-19 worries, the use and 150 perceived utility of RECHARGE stress management strategies) at baseline (T1). They will then be randomized into one of the two arms and receive either RECHARGE (intervention) or information on stress management (ATAU). RECHARGE has a typical duration of two to

153 four weeks but can take longer in case of difficulties in scheduling the intervention

154 appointments. The maximal duration between the completion of the baseline measures (T1) 
and the completion of RECHARGE is four weeks. An online questionnaire assessing the same variables as at T1 will again be completed four weeks (T2), 12 weeks (two-months follow up; T3), and 28 weeks (six-months follow-up; T4) after T1. Independence of assessments will be guaranteed by virtue of online testing. Each participant will receive an email from the trial coordinator that will contain a link to the assessment questionnaires.

\section{Randomization}

Randomization will be performed by entering the participants' ID in the order of their enrolment into a computerized list, which randomly assigns the participants to one of the two study arms. The list is generated off-site at the University of New South Wales (Australia), which is not involved in treatment delivery or data collection.

\section{RECHARGE}

The intervention consists of 4 sessions, each comprising 60 minutes, with a minimal time interval of 2 days between the sessions. The sessions will be delivered online via Skype for Business by coaches, who are trained peers (i.e., physicians, nurses, psychologists, and psychiatrists). While physician-coaches will only coach physicians and nurse-coaches only nurses, psychologist- and psychiatrist-coaches can deliver the intervention to all HCWs. Prior to the intervention, the coach will call the participant to introduce the program and schedule the sessions. The four sessions are structured as follows:

- Session 1. Psychoeducation and managing stress: Psychoeducation aims to inform participants about common emotional and physical reactions to distressing situations at work and/or during the pandemic. It thus helps the participants to understand, normalize and reduce their reactions to the current situation. In addition, a brief and 
simple arousal reduction technique (slow breathing) will be taught to enhance relaxation in the face of acute stressors.

- Session 2. Managing worries: This strategy helps participants to categorise their session. worries into solvable and unsolvable problems. It is a new strategy that is not part of $\mathrm{PM}+$ and it was thought to be important for this particular population facing pandemic-related adversity. Participants are taught to address solvable worries with problem solving techniques. Alternatively, for unsolvable worries, participants are taught a combination of mindfulness and cognitive techniques to postpone their worries. This strategy supports participants in managing their worries.

- Session 3. Meaningful activities: By using behavioral activation, this strategy is aimed at re-engaging participants gradually with meaningful activities that give them a sense of pleasure, achievement, and social connection. This strategy aims to improve participants' level of activity by incorporating attention training to enhance their mood and functioning.

- Session 4. Staying well: In this last session, the coaches provide education on how to maintain gains made and prevent a relapse. They help participants to identify foreseeable stressful situations and develop a plan to cope with them. Moreover, participants identify goals for the future and ways of achieving them. Finally, participants, consider how to extend their learning from the program in other areas of life.

In between the sessions, participants are asked to practise the previously taught strategies at home with the help of handouts. Their progress will be reviewed during the following 
204 Coaches that will deliver RECHARGE will meet the following criteria:

205

206

$-\quad \geq 18$ years of age

- German-speaking

- Working as HCW in Switzerland

- Having a completed education in medicine, nursing, psychology or any other profession within health care.

Coaches in this study will receive two days training by WHO certified Problem Management Plus master trainers who have adapted Problem Management Plus to RECHARGE. As a further part of their training, coaches are required to deliver the intervention to two mock participants and be a mock participant for the intervention themselves. Adherence to the RECHARGE manual will be ensured by the master trainers, who will watch a video-taped intervention (i.e., all four sessions) with one of the mock participants and, using a checklist to ensure basic elements of the RECHARGE intervention have been followed as required.

Moreover, the coaches will receive one-hour weekly group supervision by the master trainers, the frequency of which will be reduced gradually over the duration of the study, depending on perceived competency of the coaches.

\section{Active Treatment-as-Usual (ATAU)}

In the ATAU condition, HCWs will be referred to two recommended webpages that outline adaptive, well-validated coping strategies for managing distress; one is from the University Hospital Zurich and the other from the Mental Health Network Switzerland (Netzwerk Psychische Gesundheit Schweiz) operated by the Swiss government. Participants are asked to consult these webpages and follow instructions accordingly whenever they feel distressed.

\section{Screening measures}


230 Distress: The Kessler Psychological Distress Scale - K-10 [12, 13], a brief screening

231 questionnaire assessing general psychological distress will be used. It consists of ten items

232 (e.g., "During the last 30 days, about how often did you feel tired out for no good reason?")

233 rated on a 5-point Likert scale $(1=$ none of the time, $5=$ all of the time $)$. The questionnaire

234 will be adapted to refer to the past two weeks.

235

236

Secondary outcomes

237 Worries: The Generalized Anxiety Disorder 7 [GAD-7; 15] is a 7-item self-report

238 questionnaire that measures how often during the past two weeks the participants have been

239 bothered by problems involving worry. Items are answered on a 4-point Likert scale $(0=n o t$

240 at all, 3 = nearly every day), with a higher score indicating higher levels of worry.

241 Anxiety and depression: The Hospital Anxiety and Depression Scale [HADS; 16] is a well

242 validated and widely used 14-item scale measuring symptoms of anxiety and depression (e.g.,

243 "I feel tense or wound up"). A higher score indicates a higher symptom level of anxiety and 244 depression.

245 Burnout: The Maslach Burnout Inventory - Health Services Survey [MBI-HSS; 17] is

246 considered the gold standard in measuring burnout in HCWs. West and colleagues [18, 19]

247 have validated a two-item screening instrument in physicians including "I feel burned out

248 from my work" and "I have become more callous toward people since I took this job" $(0=$

249 never, 6 =daily), which will be used in the present study. A higher score indicates a higher

250 level of burnout.

251 Posttraumatic stress: A 4-items short form of the Posttraumatic Stress Disorder (PTSD)

252 Checklist [PCL-5; 20] that measures PTSD symptoms corresponding to the DSM-5 criteria 
will be used. Items (e.g., "Feeling distant or cut off from other people?") are measured on a 5point Likert scale $(0=$ not at all, $4=$ extremely $)$.

255

Distress due to perceived ethical dilemmas: The 4 items short form of the Moral Injury Appraisals Scale [MIAS; 21] will be included to assess distress as a consequence of acting or being witness to others acting against one's own moral rules (e.g., "I am troubled because I acted against important moral rules"). Items are answered on a 4-point Likert scale ( $1=$ not at all, 4 = very much), with a higher score indicating a higher level of distress.

Work performance: The Work Ability Index [WAI; 22, 23] assesses the self-perceived work ability and productivity with seven items (e.g., "Assume that your work ability at its best has a value of 10 points. How many points would you give your current work ability?" $(0=$ completely unable to work, $10=$ best work ability at present). Item 3 (disease catalogue) and item 7 (mental well-being) were not included in the study. Item 7 was deemed redundant because of other measurements that assess mental well-being, while the time frame used in item 3 is not applicable to this study and the item was already partially integrated into one exclusion criteria. A higher score indicates a higher work ability.

All questionnaires except for the PCL-5 (past month) will be adapted to refer to the past two weeks.

\section{Other outcomes}

We developed other single items in order to measure: work hours ("At the moment, how many hours per week do you work?”), sleep hours (“On average, how many hours per night did you sleep during the past two weeks?"), shift work ("Did you work shifts during the past two weeks?"), and COVID-19 worries (“At the moment, do you feel stressed by the COVID19 pandemic?”, "I have worries regarding COVID-19. These items are answered on 5-point scales $(0=$ not at all, 4 = very strongly $)$. We developed a 9-item scale - Reducing Tension 
Checklist (RTC) - to measure the use and perceived utility of the various strategies that are

279

280

281

282

283

284

285

286

287

288

289

290

291

292

293

294

295

296

297

298

299

300

301

trained during the intervention.

Analysis

All analyses will be conducted using $R$ and SPSS software. For the RCT, both intention-totreat (ITT) analysis and completers' (per protocol) analyses will be carried out. The primary analysis will be the ITT analysis. Different trajectories of outcomes will be analyzed using mixed linear modelling, defining treatment as fixed effects, baseline measurement of primary endpoint as covariate, and subject as random effects. The benefit of this approach is that it presumes intent-to-treat analyses as hierarchical linear modelling allows the number of observations to vary between participants and effectively handles missing data. Time, treatment condition, and their interaction will be included in the models. Baseline measures (e.g. age, gender, levels of worries, depression, anxiety, etc.) will be used as covariates to determine predictors of treatment response for each arm. Results will be presented as differences in mean change on the K-10 (primary outcome) and on all other variables (secondary outcomes) from baseline to T3 compared between RECHARGE and ATAU groups, with $95 \%$ confidence intervals and p-values. In addition, differences in mean change on all variables will also be calculated from baseline to $\mathrm{T} 2$ and $\mathrm{T} 4$.

\section{Adverse events reporting - Data and Safety Monitoring Committee}

Adverse events will be reported according to the Swiss Human Research Ordinance. In addition, based on the principles set out by Ellenberg and colleagues [24], adverse events will be reported to the Data and Safety Monitoring Board (DSMB), comprising an independent clinical specialist, statistician, and trial methodologist. Stopping or modifying the trial for 
safety will consider the balance of ensuring safety and how stopping will impact clinical practice.

\section{Discussion}

306 In times of crises such as the current COVID-19 pandemic, HCWs can face many additional 307 stressors compared to normal circumstances. These include higher work demands, fear of contagion, and social isolation. Psychological interventions are needed to address complex stressors put on HCWs during times of crises and support them in their stress management. Importantly, such interventions have to deal with extraordinary circumstances due to the

311 crisis, which, in the case of the COVID-19 pandemic, include home confinement, social

312 distancing, and workloads potentially much higher than usual. This necessitates online 313 delivery, high flexibility, and minimal time costs of the intervention. Moreover, as many

314 HCWs are affected by adversities at the same time, such an intervention needs to be easily scalable. RECHARGE accommodates these demands as its strategies are simple and well validated in times of crises $[11,25]$, its delivery is brief, flexible in terms of time and

317 personnel needed, and fully online accessible. Moreover, training of coaches is brief and does not require mental health specialists, which improves scalability of the intervention. Therefore, we believe that this program will deliver a much-needed template for an intervention to reduce stress and enhance coping in HCWs in times of crises. RECHARGE could help to ensure HCW well-being, and uphold the performance of the workforce that is vital to deal with the overload posed upon the health care system by virus outbreaks. If proven effective, RECHARGE may not only be used in Switzerland to support the response of HCWs to the current pandemic, but also globally and for other crises. In addition, it may be helpful under non-pandemic circumstances within health care, or could be used as a program to enhance resilience to stress in preparation of pandemics. Finally, it may be 
adapted so that a variety of populations with increased psychological distress (e.g., other hospital staff or individuals working in other areas) can benefit from the intervention. RECHARGE may even be tested in different formats (e.g., group version).

\section{Trial Status}

332 The study is currently recruiting and enrolling participants. Recruitment began on August 28, 2020, and the approximate date for completion of recruitment will be June 30, 2021.

\section{Ethical consideration and informed consent}

336 As confirmed and reviewed by the Cantonal Ethics Committee of Zurich (Switzerland), this study does not fall within the scope of the Human Research Act (BASEC-NR: 2020-00796). Therefore, an authorization from the ethics committee was not required. Nevertheless, this study was conducted according to the Swiss Human Research Ordinance (i.e., under strict confidentiality and privacy, with coding of health-related personal data) and the criteria set

341 by the declaration of Helsinki. All participants receive written information on the nature, purpose and procedure of the project, their right to withhold or revoke their consent at any time, and their right to receive information. All participants give their informed consent by

344 checking the consent box in our online study information and informed consent form prior to 345 any study-related procedures.

\section{Consent for publication}

348 Not applicable. 
351 This study follows open science practices and will use open access journals and repositories

352 to publish results.

353 The data will be available from the author on reasonable request.

354

355 Clinical Trial Registration

356 This study has been preregistered in the Clinical Trials Registry of the U.S. National Library

357 of Medicine on August 26, 2020 (CT04531774).

358

359 Competing interests

360 The authors declare that they have no competing interests.

361

362 Author's contributions

363 All authors were involved in the concept and design of the study. KSD, NM, RB and SW

364 developed the RECHARGE manual. NM and SW drafted the study protocol for ethics review

365 and for the manuscript, and all authors commented and approved of the final versions.

366

367 Funding 
368 The study is funded by USZ Foundation and Klaus-Grawe-Foundation, Zurich, Switzerland.

369 The funding foundations were not involved in the design of the study, collection, analysis,

370 and interpretation of data and in writing the manuscript.

371

372 Acknowledgments

373 We thank Aiysha Malik from World Health Organization (WHO), Department of Mental

374 Health and Substance Abuse, Geneva, Switzerland for her valuable comments on the study

375 and this paper. We also thank the Coaches and all participating HCWs who, despite their

376 workload, participated in this study.

377

378 The authors alone are responsible for the views expressed in this article, which do not

379 necessarily represent the views, decisions or policies of the institutions with which they are

380 affiliated.

381 
1. Committee on Systems Approaches to Improve Patient Care by Supporting Clinician, W.B., et al., Taking Action Against Clinician Burnout: A Systems Approach to Professional Well-Being. 2019, Washington, D.C.: National Academies Press.

2. Kisely, S., et al., Occurrence, prevention, and management of the psychological effects of emerging virus outbreaks on healthcare workers: rapid review and meta-analysis. BMJ, 2020: p. m1642.

3. Pappa, S., et al., Prevalence of depression, anxiety, and insomnia among healthcare workers during the COVID-19 pandemic: A systematic review and meta-analysis. Brain, Behavior, and Immunity, 2020: p. S088915912030845X.

4. World Health Organization, W., Health workforce policy and management in the context of the COVID-19 pandemic response. 2020.

5. Shanafelt, T.D., J. Ripp, and M. Trockel, Understanding and Addressing Sources of Anxiety Among Health Care Professionals During the COVID-19 Pandemic. JAMA, 2020.

6. Regehr, C., et al., Interventions to Reduce the Consequences of Stress in Physicians: A Review and Meta-Analysis. The Journal of Nervous and Mental Disease, 2014. 202(5): p. 353-359.

7. West, C.P., et al., Interventions to prevent and reduce physician burnout: a systematic review and meta-analysis. The Lancet, 2016. 388(10057): p. 2272-2281.

8. $\quad$ Petrie, K., et al., Interventions to reduce symptoms of common mental disorders and suicidal ideation in physicians: a systematic review and meta-analysis. Lancet Psychiatry, 2019. 6(3): p. 225-234.

9. Serrano-Ripoll, M.J., et al., Impact of viral epidemic outbreaks on mental health of healthcare workers: a rapid systematic review and meta-analysis. J Affect Disord, 2020. 277: p. 347-357.

10. Dawson, K.S., et al., Problem Management Plus (PM+): a WHO transdiagnostic psychological intervention for common mental health problems. World Psychiatry, 2015. 14(3): p. 354-7.

11. Bryant, R.A., et al., Effectiveness of a brief behavioural intervention on psychological distress among women with a history of gender-based violence in urban Kenya: $A$ randomised clinical trial. PLoS Med, 2017. 14(8): p. e1002371.

12. Kessler, R.C., et al., Screening for serious mental illness in the general population. Archives of General Psychiatry, 2003. 60(2): p. 184-189.

13. Kessler, R.C., et al., Short screening scales to monitor population prevalences and trends in non-specific psychological distress. Psychological Medicine, 2002. 32(6): p. 959-976.

14. Rahman, A., et al., Effect of a Multicomponent Behavioral Intervention in Adults Impaired by Psychological Distress in a Conflict-Affected Area of Pakistan: A Randomized Clinical Trial. JAMA, 2016. 316(24): p. 2609-2617.

15. Spitzer, R.L., et al., A Brief Measure for Assessing Generalized Anxiety Disorder: The GAD-7. Archives of Internal Medicine, 2006. 166(10): p. 1092.

16. Snaith, R.P., et al., Hospital anxiety and depression scale: deutsche Version ; HADS-D ; ein Fragebogen zur Erfassung von Angst und Depressivität in der somatischen Medizin ; Testdokumentation und Handanweisung. 1. Aufl ed. 1995, Bern: Huber. 43.

17. Maslach, C., S.E. Jackson, and M.P. Leiter, Maslach burnout inventory manual. 4th edition ed. 2018, Menlo Park, CA: Mind Garden.

18. West, C.P., et al., Concurrent Validity of Single-Item Measures of Emotional Exhaustion and Depersonalization in Burnout Assessment. Journal of General Internal Medicine, 2012. 27(11): p. 1445-1452.

19. West, C.P., et al., Single Item Measures of Emotional Exhaustion and Depersonalization Are Useful for Assessing Burnout in Medical Professionals. Journal of General Internal Medicine, 2009. 24(12): p. 1318-1321. 
433

434

435

436

437

438

439

440

441

442

443

444

445

446

447

20. Price, M., et al., Investigation of abbreviated 4 and 8 item versions of the PTSD Checklist 5. Psychiatry Research, 2016. 239: p. 124-130.

21. Hoffman, J., et al., The relationship between moral injury appraisals, trauma exposure, and mental health in refugees. Depression and Anxiety, 2018. 35(11): p. 1030-1039.

22. Hasselhorn, H.M. and G. Freude, Der Work Ability Index: ein Leitfaden. Schriftenreihe der Bundesanstalt für Arbeitsschutz und Arbeitsmedizin Sonderschrift. 2007, Bremerhaven: Wirtschaftsverl. NW, Verl. für neue Wiss. 54.

23. Netzwerk, I.W., Wie steht es um Ihre Arbeitsfähigkeit? WAI*-Fragebogen \& Auswertung (Kurzversion). 2017.

24. Ellenberg, S.S., R.T. Fleming, and L.D. DeMets, Data Monitoring Committees in Clinical Trials. A Practical Perspective. 2002, West Sussex: Wiley.

25. Rahman, A., et al., Effect of a Multicomponent Behavioral Intervention in Adults Impaired by Psychological Distress in a Conflict-Affected Area of Pakistan: A Randomized Clinical Trial. JAMA, 2016. 


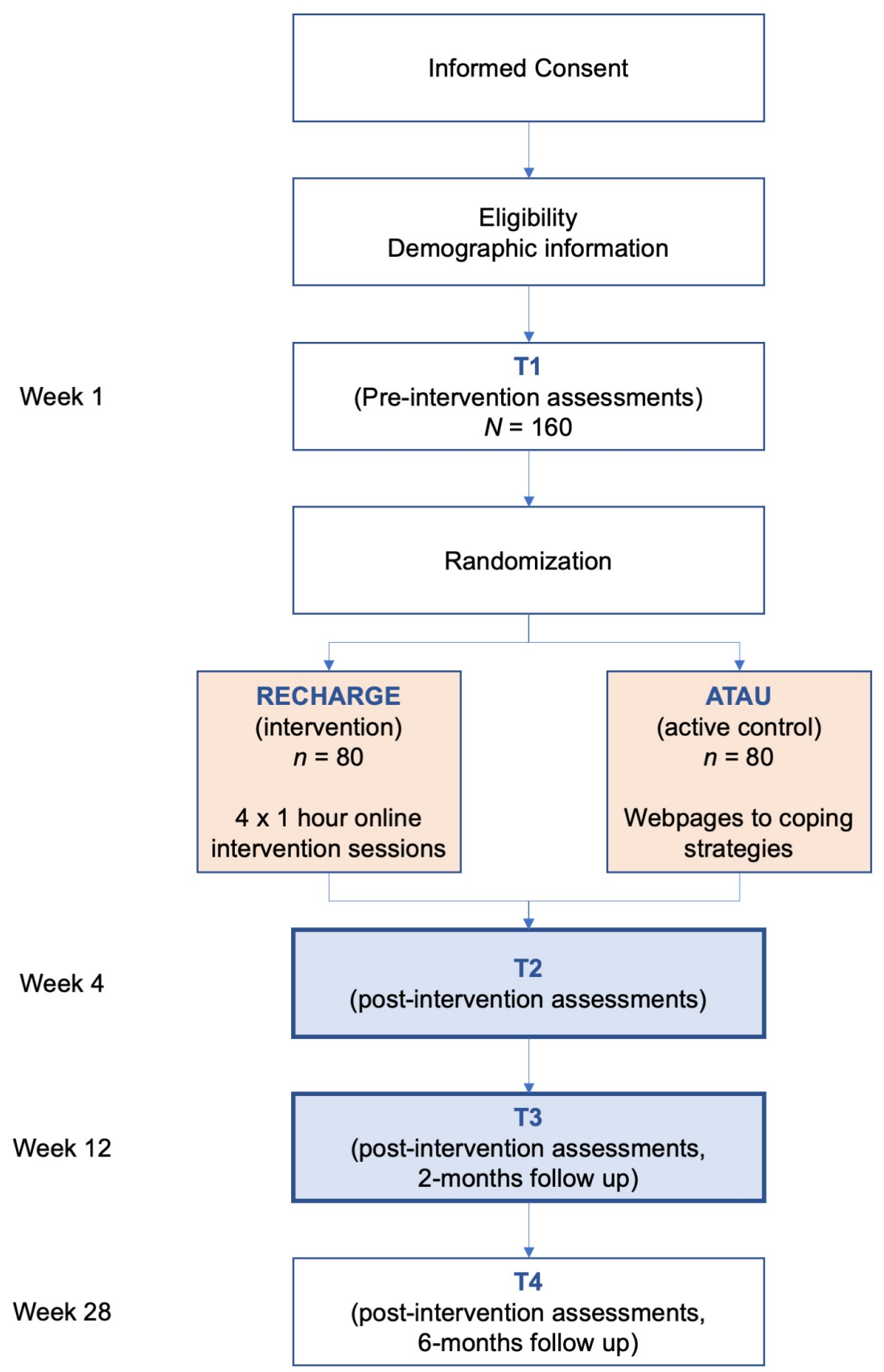

449

450 Figure 1: Flow diagram 
STUDY PERIOD

\begin{tabular}{|c|c|c|c|c|c|c|c|}
\hline \multirow[b]{3}{*}{ Timepoint } & \multicolumn{3}{|c|}{ Enrolment } & \multicolumn{4}{|c|}{ Post Allocation } \\
\hline & \multirow{2}{*}{$\begin{array}{l}\text { Registration } \\
\text { T0a (Week } \\
\text { 0) }\end{array}$} & \multicolumn{2}{|c|}{ Allocation } & \multirow{2}{*}{$\begin{array}{l}\text { Baseline } \\
\text { T1 (Week 1) }\end{array}$} & \multirow{2}{*}{$\begin{array}{l}\text { Postmeasurement } \\
\text { T2 (Week 5) }\end{array}$} & \multirow{2}{*}{$\begin{array}{l}2 \text { month Follow } \\
\text { Up } \\
\text { T3 (Week 13) }\end{array}$} & \multirow{2}{*}{$\begin{array}{l}6 \text { month Follow } \\
\text { Up } \\
\text { T4 (Week 29) }\end{array}$} \\
\hline & & T0b (Week 0) & T0c (Week 0) & & & & \\
\hline \multicolumn{8}{|l|}{ Enrolment: } \\
\hline Personal Data & $\mathrm{X}$ & & & & & & \\
\hline Informed Consent & $\mathrm{X}$ & & & & & & \\
\hline Eligibility Screen & & $\mathrm{X}$ & & & & & \\
\hline Occupational Data & & $\mathrm{X}$ & & & & & \\
\hline Randomization & & & $\mathrm{X}$ & & & & \\
\hline \multicolumn{8}{|l|}{ Interventions: } \\
\hline \multicolumn{8}{|l|}{ RECHARGE } \\
\hline ATAU & & & & $\longleftarrow$ & $\Rightarrow$ & & \\
\hline \multicolumn{8}{|l|}{ ASSESSMENTS: } \\
\hline $\mathrm{K}-10$ & & $\mathrm{X}$ & & $\mathrm{X}$ & $\mathrm{X}$ & $\mathrm{X}$ & $\mathrm{X}$ \\
\hline GAD-7 & & & & $X$ & $X$ & $X$ & $X$ \\
\hline HADS & & & & $\mathrm{X}$ & $\mathrm{X}$ & $\mathrm{X}$ & $\mathrm{X}$ \\
\hline MBI-HSS & & & & $\mathrm{X}$ & $\mathrm{X}$ & $\mathrm{X}$ & $\mathrm{X}$ \\
\hline PCL-5 & & & & $\mathrm{X}$ & $\mathrm{X}$ & $\mathrm{X}$ & $\mathrm{X}$ \\
\hline MIAS & & & & $\mathrm{X}$ & $\mathrm{X}$ & $\mathrm{X}$ & $\mathrm{X}$ \\
\hline WAI & & & & $\mathrm{X}$ & $\mathrm{X}$ & $\mathrm{X}$ & $\mathrm{X}$ \\
\hline
\end{tabular}

451

452

Table 1: SPIRIT Overview 


\section{Figures}

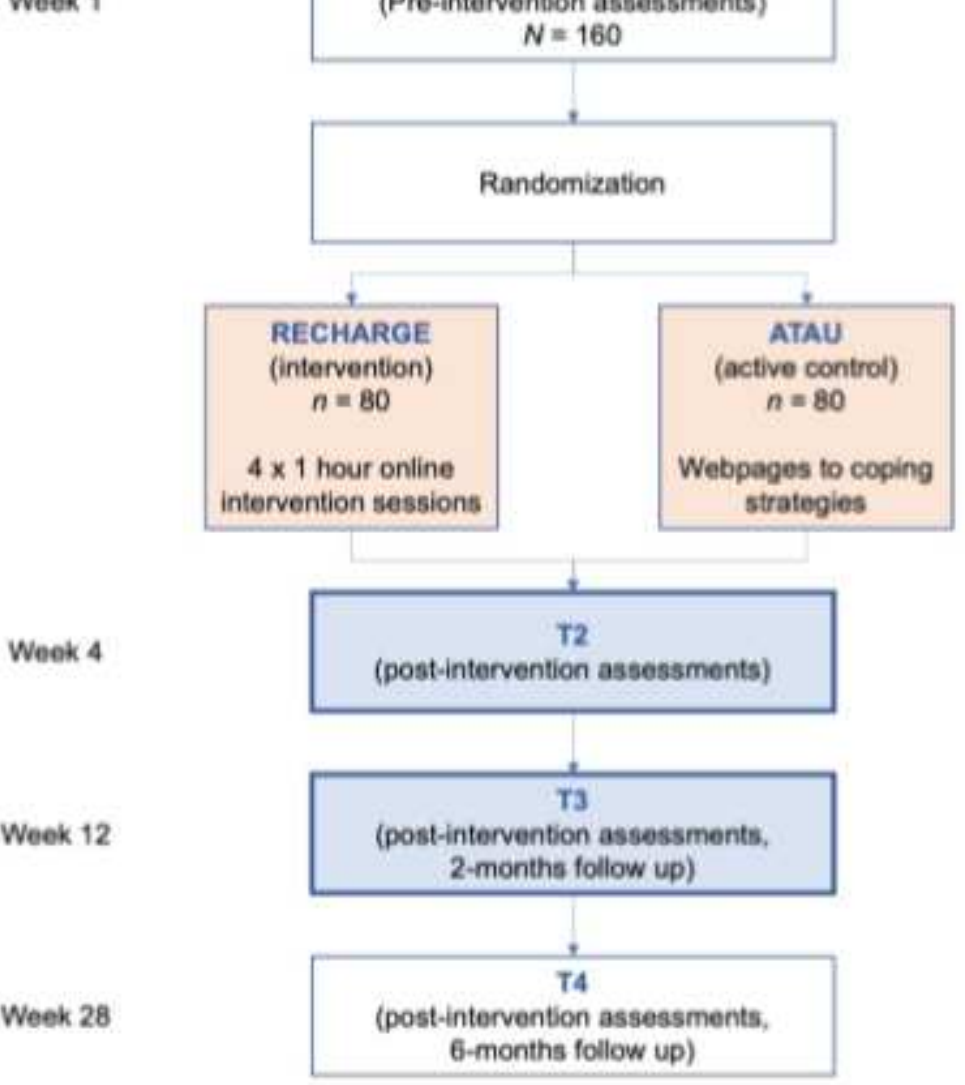

Figure 1

Flow diagram

\section{Supplementary Files}

This is a list of supplementary files associated with this preprint. Click to download.

- RECHARGESPIRITCheckliste.pdf 